\title{
Validación de un inventario de roles de masculinidad hegemónica en varones colombianos*
}

\section{Validation of an inventory of hegemonic masculinity roles in Colombian men}

Carlos Alejandro Pineda-Roa** ORCID:0000-0002-9000-9302

Universidad del Norte, Colombia

Geydi Katherine Galindo-Ascanio ORCID: 0000-0002-7852-3843 Universidad Pedagógica y Tecnológica de Colombia

Diana Paola González-Moreno ORCID: 0000-0002-4945-0489 Universidad Pedagógica y Tecnológica de Colombia

Reynel Alexander Chaparro-Clavijo ORCID: 0000-0003-2288-1542 Universidad Nacional de Colombia

Recibido: 6 diciembre de 2018 Revisado: 16 de febrero de 2019 Aceptado: 27 de marzo de 2019

\section{Resumen}

El objetivo del presente estudio consistió en evaluar las propiedades psicométricas de un inventario para medir el concepto de masculinidad hegemónica en varones residentes en una ciudad colombiana intermedia. Se seleccionaron mediante muestreo no probabilístico por disponibilidad 600 participantes con edades comprendidas entre 18 y 65 años $(M=29$, $D E=10.7)$. Se aplicó un inventario de 30 ítems que mide la adherencia a los roles de masculinidad hegemónica versión modificada (IRMH-M). El Análisis Factorial Exploratorio (AFE) arrojó cinco factores que explican el $64 \%$ de la varianza común. El Análisis Factorial Confirmatorio (AFC) confirmó muy buen ajuste de este modelo pentafactorial en una versión reducida de 17 ítems S-B $x^{2}=343.29, \mathrm{gl}=109$, NNFI =0.97; $\mathrm{CFI}=0.98$. RMSEA $=0.06 \mathrm{IC} 95 \%$ (0.053 - 0.067). LoS coeficientes Alfa y Omega obtenidos para estimar consistencia interna estuvieron entre 0.70 y 0.79 . El constructo "roles de masculinidad hegemónica"

Artículo de investigación. Artículo derivado del proyecto Estudio latinoamericano de bienestar psicológico, calidad de vida y riesgo de trastornos de alimentación en varones gay de diez países. Cómo citar: Pineda-Roa, C. A., Galindo-Ascanio, G. K., González-Moreno, D. P., \& ChaparroClavijo, R. A. (2019). Validación de un Inventario de roles de masculinidad hegemónica en varones colombianos. Diversitas: Perspectivas en Psicología, 15(2), 257-270. DOI: 10.15332/22563067.5401

Correspondencia: Carlos Alejandro Pineda, Agradecimientos a Colciencias por financiación Doctorado en Psicología en la Universidad del Norte, Barranquilla (convocatoria 727 de 2015). pinedaac@uninorte.edu.co. Correos electrónicos: Geydi Katherine Galindo-Ascanio, Correspondencia: geydi.galindo@uptc.edu.co; Diana Paola González-Moreno, diana.gonzalezmoreno@uptc.edu.co; Reynel Alexander Chaparro-Clavijo, Agradecimientos a Colciencias por financiación de Doctorado en Psicología en la Universidad Nacional de Colombia (convocatoria 647 de 2014 ). rachaparroc@unal.edu.co. 
discriminó adecuadamente según la edad, el nivel educativo, el estrato y la ocupación. Se concluye que IRMH-M posee propiedades psicométricas adecuadas.

Palabras clave: rol de género, masculinidad hegemónica, estudios de validación.

\begin{abstract}
The aim of this study was to evaluate the psychometric properties of an inventory to measure the hegemonic masculinity in men residing in an intermediate city in Colombia. A sample of 600 participants between 18 to 65 years old were selected using a non-probabilistic sampling for availability $(M=29, S D=10.7)$. A 30 items inventory that measure the adherence to the roles of hegemonic masculinity modified version MNRI-M was applied. Exploratory Factor Analysis EFA showed five factors that explain $64 \%$ of the common variance. Confirmatory Factor Analysis CFA confirmed very good fit of this penta-factorial model in a reduced version of 17 items S-B $x^{2}=343.29, \mathrm{gl}=109, \mathrm{NNFI}=0.97$; $\mathrm{CFI}=0.98$. RMSEA $=0.06 \mathrm{IC} 95 \%(0.053,0.067)$. Internal consistency, Alpha and Omega coefficients, were between 0.70 and 0.79 . Constructed roles of hegemonic masculinity discriminated adequately according to age, educational level, socioeconomic classification, and occupation. It is concluded that MNRI-M has adequate psychometric properties.
\end{abstract}

Keywords: gender role, hegemonic masculinity, validation studies.

\section{Introducción}

El género es una variable que cobra cada vez más importancia en el campo de la salud pública (Estrada \& Sánchez-Alfaro, 2011). Así mismo, se reconoce la necesidad de vincular a los hombres en relaciones más equitativas (Organización Panamericana de la Salud [OPS], 2010). La masculinidad hegemónica ha tomado relevancia, en cuanto se considera un componente esencial para explicar la violencia estructural y la salud pública (Scott-Samuel, Stanistreet, \& Crawshaw, 2009).

Históricamente, el estudio del género se ha centrado en las mujeres, desdibujando la importancia de abordar lo que sucede alrededor de los hombres (Burin \& Meler, 2000). Por esto, el presente estudio busca incorporar únicamente varones. Las perspectivas socioconstruccionistas comprenden las masculinidades como una construcción que se aprende y se practica en el devenir cultural, histórico y social, por lo que no son estáticas, ni son las mismas siempre y en todas partes (Faur, 2004). Siguiendo a Hernández (2008), “es, más bien, la masculinidad que ocupa la posición privilegiada en un modelo prescriptivo dado de relaciones de género, una posición siempre en disputa" (p.125).

Cuestionar el rol de género tradicional se considera una amenaza al privilegio y poder social del hombre heterosexual y a la masculinidad hegemónica. En este sentido, se puede comprender que los hombres homosexuales sean percibidos de forma más negativa que las mujeres homosexuales. Por lo tanto, las diferencias de género no están dadas por el sexo biológico sino por las actitudes del rol de género que se desarrollan a través del proceso de socialización y crianza (Cruz, 2007; Kerns \& Fine, 1994).

Una masculinidad hegemónica puede relacionarse con el mantenimiento de una estructura social autoritarita que incluye los roles de género tradicionales como la superioridad de los hombres sobre las mujeres y que aquellos que no cumplen con este criterio sean considerados débiles o femeninos como los homosexuales (Cruz, 2007). Un análisis de varios discursos de figuras de poder en Colombia y de normatividades que penalizaban la homosexualidad, encuentra consistencias de la expresión de la masculinidad hegemónica, que se recalca en el 
mantenimiento de una estructura social heteronormada (Bustamante, 2013).

Esta estructura de poder somete a otras expresiones de la masculinidad que no se ajustan al modelo de comportamiento, valores y prácticas propios de la masculinidad hegemónica. Análisis de los ingresos económicos de diferentes grupos de hombres, encuentran que aquellos con bajos ingresos tienen menores oportunidades en educación, en el empleo formal y la salud. Estas situaciones afectan condiciones como el ser proveedor de la familia, contar con dinero para trabajar un cuerpo que se parezca al modelo social dominante de belleza masculina y, entre otros, sufren el rechazo, la discriminación y la violencia por parte de otros hombres de sectores más altos quienes son generalmente sus patrones $\mathrm{y}$ los explotan laboralmente (Bard, 2016).

La presión que pueden sentir algunos hombres para adquirir o acercarse al estándar de la masculinidad hegemónica trae diferentes consecuencias, como: una baja autoestima (Davis, 1987), depresión (Good \& Mintz, 1990) y violencia de pareja (Jakupcak, Lisak, \& Roemer, 2002). Levant y Richmond (2007) advierten que los hombres exhiben mayor arraigo a los roles de masculinidad hegemónica que las mujeres. En efecto, intervenciones para disminuir el malestar de la masculinidad hegemónica en los varones se orientan a un proceso reflexivo que la cuestione, al tiempo que se proponen alternativas para explorar y empoderarles en trabajar por la equidad de género; por ejemplo, cuando la masculinidad hegemónica es un elemento importante de la comprensión de la violencia (Jewkes et al, 2015) y el acceso a cuidados en salud (Torres-Pagán \& Toro-Alfonso, 2017).

\section{Antecedentes de medición de masculinidad hegemónica}

David y Brannon (1976) definieron cuatro factores de la masculinidad hegemónica que incluyen: evitación de lo femenino, tener éxito y logros continuamente, no mostrar signos de debilidad y buscar la aventura incluso a riesgo de violencia. Bajo estos supuestos se desarrolló la escala de masculinidad Brannon Masculinity Scale (Brannon \& Juni, 1984).
Posteriormente, para evitar la redundancia entre escalas e incluyendo el rechazo y el odio hacia personas homosexuales y las actitudes hacia el sexo, se propone la primera versión del Male Role Norms Inventory (MRNI) (Levant, Wu, \& Fischer, 1996) que incluye la medición de la masculinidad hegemónica y la no hegemónica en ocho factores: evitar la feminidad, actitudes hacia homosexuales, la autosuficiencia, la agresión, el logro/estatus, las actitudes hacia el sexo, la emocionalidad restrictiva y un factor de masculinidad no hegemónica.

Ajustes posteriores del MRNI han identificado subescalas con baja confiabilidad, como es el caso del factor de masculinidad no hegemónica (Levant et al., 2007), y la reducción de ítems (Levant, Hall \& Rankin, 2013) en los cuales se han eliminado ítems iniciales del MRNI al tiempo que se han añadido otros. Otras versiones se han desarrollado y ajustado para comprender cómo se establecen roles de género en la adolescencia, por ejemplo, el MRNI-A (Brown, 2002; Levant, Graef, Smalley, Williams, \& McMillan, 2008).

El concepto de masculinidad en general se ha trabajado en Latinoamérica desde diferentes ciencias sociales, incluida la psicología, en el que ha predominado para su análisis el uso de metodologías cualitativas (Hernández, 2008; Vázquez \& Castro, 2009). También se reconocen estudios psicométricos que han abordado la masculinidad hegemónica de forma indirecta, por ejemplo, aplicando escalas de expresividad y homofobia (Lozano \& Rocha, 2011) y de forma directa a través de adaptaciones de la escala de roles de masculinidad de Levant y Fischer (Toro-Alfonso, Walters-Pacheco, \& Sánchez, 2012). No obstante, reconocemos que la investigación que utiliza medidas objetivas es aún escasa, por lo tanto, con un gran potencial de desarrollo en el área de las masculinidades.

El abordaje de la medición de las masculinidades en países latinoamericanos se ha desarrollado desde distintas perspectivas teóricas. Por ejemplo, en México se desarrolló el IMAFE (Inventario de Masculinidad y Feminidad) (Lara, 1993) el cual, a su vez fue validado en adolescentes y jóvenes colombianos (Martínez-Gómez, Guerrero-Rodríguez, \& Rey-Anacona, 2012) y evalúa los roles de género 
como un rasgo más de la personalidad. En la misma línea teórica de Lara (2003), Barra (2004) construyó un inventario para evaluar roles sexuales basado en la teoría de los esquemas de Bem (1981), el cual mide roles típicamente femeninos (expresivos) y típicamente masculinos (instrumentales) y otros roles indiferenciados y andróginos. A pesar de que estos trabajos reflejan un avance importante, mantienen una postura binaria del género y no evalúan explícitamente roles de género propios de orientaciones sexuales no normativas u homosexuales. Así, el inventario de roles de masculinidad hegemónica IRMH traducido del MRNI se convierte en una alternativa muy promisoria.

Además de lo señalado anteriormente, se identificó que el IRMH-M es la única versión a la fecha, traducida y adaptada al español del MRNI (ToroAlfonso \& Varas-Díaz, 2003). Por lo tanto, es necesario evidenciar su comportamiento psicométrico en diferentes poblaciones y contextos, para mostrar las potencialidades en el análisis de la masculinidad hegemónica en estudios que involucran a hombres hispanohablantes. El presente estudio busca realizar un análisis psicométrico del IRMH-M en una muestra de varones residentes en la ciudad de Tunja (Colombia).

\section{Método}

\section{Diseño}

La presente investigación es de carácter psicométrico de tipo instrumental, debido a que estuvo encaminada al desarrollo de pruebas y aparatos, incluyendo tanto el diseño o adaptación, como el estudio de las propiedades psicométricas de los mismos (Montero \& León, 2007), pretendiendo validar el IRMH-M a la población de hombres residentes en la ciudad de Tunja (Colombia).

\section{Participantes}

Participaron 600 hombres residentes en la ciudad de Tunja (Colombia), con edades que oscilaron entre los 18 y $65(M=29, D T=10.75)$. Los participantes del presente estudio fueron seleccionados a través de muestreo no probabilístico por disponibilidad. Se establecieron como criterios de inclusión el ser mayores de edad y la firma del consentimiento informado.

\section{Consideraciones éticas}

Acorde con las normas colombianas para la investigación en ciencias de la salud, se obtuvo consentimiento informado a cada participante (Resolución 8430 de 1993 del Ministerio de Salud, 1993). La participación fue voluntaria y quedó explícito que la investigación no generaría beneficios directos sino indirectos en cuanto a recopilar información relevante que aporte indicios sobre el desempeño psicométrico de pruebas psicológicas. En dicho consentimiento se garantizó la confidencialidad al no solicitar datos personales como número de teléfono o direcciones físicas o electrónicas. Por último, con la firma del consentimiento, los participantes avalaron la publicación de los resultados.

\section{Instrumento}

El IRMH-M de Toro-Alfonso y Varas-Díaz (2003) es una versión corta de 30 ítems del MRNI de Levant, Wu y Fischer (1996) el cual constaba de 57 ítems. Toro-Alfonso y Varas-Díaz (2003) lo adaptaron a la población puertorriqueña con un único cambio de sentido en el ítem "when the going gets tough, men should get tough" el cual quedó en la versión en español como "un hombre debe ser fuerte en los momentos difíciles". Con el argumento de evitar la redundancia y la baja confiabilidad, se suprimieron dos subescalas (autoconfianza y emocionalidad restrictiva). Así, la versión de 30 ítems validada en el presente trabajo corresponde a cinco de las siete subescalas en el MRNI original denominadas: evitación de conductas femeninas, logro/estatus, agresión, rechazo a los homosexuales y actitudes hacia el sexo.

El IRMH-M fue traducido y adaptado en Puerto Rico por Toro-Alfonso y Varas-Díaz (2003) y este se aplicó originalmente en una muestra de hombres homosexuales latinoamericanos en un proyecto denominado "Estudio latinoamericano de bienestar 
psicológico, calidad de vida y riesgo de trastornos de alimentación en varones gay de diez países", liderado por Toro-Alfonso y Varas-Díaz de la Universidad de Puerto Rico y Universidad Católica del Norte en Chile respectivamente. El IRMH-M presenta ítems en forma de afirmaciones acerca de lo que un hombre debería exhibir o expresar de acuerdo con un imperativo cultural patriarcal. Posee una puntuación tipo likert (Totalmente de acuerdo $=1$, parcialmente de acuerdo $=2$, indeciso $=3$, parcialmente en desacuerdo $=4$, totalmente en desacuerdo $=5$ ) de tal forma que a menor puntaje mayor adherencia a los roles de masculinidad hegemónica. En el presente trabajo se aplicó la misma versión puertorriqueña de Toro-Alfonso y Varas-Díaz (2003).

\section{Procedimiento}

Con el objetivo de identificar si existía comprensión de los ítems en el contexto, se realizó una prueba piloto seleccionando 194 participantes, en su mayoría estudiantes de Psicología quienes dieron su consentimiento para su participación voluntaria respondiendo el IRMH-M de Toro-Alfonso y VarasDíaz (2003). El pilotaje tuvo por objeto validar la comprensión de las instrucciones y de los ítems. Sin embargo, este no sugirió cambio alguno para el instrumento final aplicado. Posteriormente, se realizó una revisión teórica sobre el constructo que se quería medir, para lo cual se consultaron las Bases de Datos, Redalyc, Scielo, Google Académico, ScienceDirect y Psycinfo. Luego se llevó a cabo la recolección definitiva de los datos en sectores comerciales, educativos, organizacionales y públicos de la ciudad de Tunja (Colombia).

\section{Análisis estadísticos}

Para establecer la viabilidad del análisis factorial exploratorio (AFE) se calculó el estadístico de Kaiser Meyer Olkin (KMO) y el estadístico de Bartlet. AFE fue implementado mediante la extracción "Factorización del eje principal" y rotación promax, reteniendo ítems con cargas superiores a .40. La confirmación de la estructura factorial se realizó mediante análisis factorial confirmatorio (AFC) utilizando software Lisrel versión 8.8. Para conocer la bondad del ajuste del AFC se calcularon la prueba chi cuadrado de Satorra-Bentler, con grados de libertad (gl) y valor de probabilidad $(\mathrm{p})$, y los coeficiente RMSEA (Root Mean Square Error of Approximation, raíz del cuadrado media del error de aproximación) e intervalo de confianza del 90\% (IC90\%), CFI (Comparative Fit Index, índice comparativo de ajuste), NNFI (No Normalized Fit Index, índice de ajuste no normalizado) y SRMS (Standardized Mean Square Residual, residuo cuadrado promedio estandarizado). Para chi cuadrado se esperó que el valor de probabilidad fuera $>5 \%$; para RMSEA y SRMR < 0.06. Para CFI y NNFI valores $>0.89$ (Morata-Ramírez, Holgado-Tello, BarberoGarcía, \& Mendez, 2015).

Se analizaron los resultados de consistencia interna de las subescalas encontradas en el AFE mediante Alfa de Cronbach y Omega de McDonald. Este último se calculó mediante el software libre $R$ con intervalos de confianza al 95\% (Dunn, Baguley \& Brunsden, 2014). Con el fin de evidenciar si el constructo roles de masculinidad hegemónica discrimina entre las distintas subpoblaciones, se realizó un procedimiento de validez discriminante que se calculó mediante la prueba no paramétrica $\mathrm{H}$ de Kruskal Wallis, una vez se evidenció que los datos no se distribuyeron normalmente $(\mathrm{K} . \mathrm{S}=.051, p=.001)$.

\section{Resultados}

Primero se presenta el análisis descriptivo de las puntuaciones del IRMH-M, así como las variables edad, estrato, nivel académico y ocupación. Luego se presentan los resultados de validez de constructo seguido por los niveles de confiabilidad por consistencia interna a través del alfa de Cronbach y el Omega de McDonald de la escala total y por subescalas. Finalmente, se presentan los resultados de validez discriminante en aquellas variables en que hubo diferencias estadísticamente significativas.

\section{Análisis descriptivo del IRMH-M}

Los puntajes de la variable adherencia hacia los roles de masculinidad hegemónica oscilaron entre 30 y $142(M=80.95 ; D E=24)$. 
Tabla 1.

Descriptivos de la muestra de varones residentes en Tunja (Colombia).

\begin{tabular}{|c|c|c|c|}
\hline Variable & Rango & $\mathrm{n}$ & $\%$ \\
\hline & Bajo & 352 & 58.7 \\
\hline \multirow[t]{4}{*}{ Roles de Masculinidad Hegemónica } & Moderado & 190 & 31.7 \\
\hline & Alto & 58 & 9.7 \\
\hline & Casado & 146 & 24.3 \\
\hline & Soltero & 324 & 54 \\
\hline \multirow[t]{6}{*}{ Estado civil } & Viudo(a) & 7 & 1.2 \\
\hline & Unión libre & 106 & 17.7 \\
\hline & Divorciado & 17 & \\
\hline & Estudiantes & 163 & 27.2 \\
\hline & Profesionales de la salud & 39 & 6.5 \\
\hline & Seguridad & 24 & 4.0 \\
\hline \multirow{8}{*}{ Ocupación } & Artistas & 7 & 1.2 \\
\hline & Comercio & 149 & 24.8 \\
\hline & Construcción & 117 & 19.5 \\
\hline & Otros profesionales & 82 & 13.7 \\
\hline & No labora actualmente & 19 & 3.2 \\
\hline & Primaria & 29 & 4.8 \\
\hline & Bachillerato & 115 & 19.2 \\
\hline & Técnico & 124 & 20.7 \\
\hline \multirow{7}{*}{ Nivel educativo } & Tecnólogo & 55 & 9.2 \\
\hline & Universitario en formación & 153 & 25.5 \\
\hline & Universitario completo & 90 & 15.0 \\
\hline & Posgrado & 33 & 5.5 \\
\hline & Ninguno & 1 & .2 \\
\hline & 1 & 35 & 5.8 \\
\hline & 2 & 232 & 38.7 \\
\hline \multirow{5}{*}{ Estrato } & 3 & 261 & 43.5 \\
\hline & 4 & 61 & 10.2 \\
\hline & 5 & 9 & 1.5 \\
\hline & 6 & 2 & 0.3 \\
\hline & $(18-25)$ & 224 & 37.3 \\
\hline \multirow[t]{2}{*}{ Edad (años) } & $(26-35)$ & 187 & 31.2 \\
\hline & $(36-65)$ & 189 & 31.5 \\
\hline
\end{tabular}

Fuente: elaboración propia.

La tabla 1 refleja las características más sobresalientes de la muestra. Se observa que la mayoría de los participantes se encuentran en un rango bajo de adherencia a los roles de masculinidad hegemónica; en su mayoría son jóvenes universitarios (25.5\%), técnicos (20.7\%) o con bachillerato terminado (19.2\%). El $52 \%$ solteros y el $82 \%$ pertenecían a estratos dos y tres.

\section{Validez de constructo.}

Los resultados del índice KMO (0.92) y el valor de significancia para la prueba de esfericidad de Bartlett $(7271.56 ; p<.001)$ señalan que la matriz de correlaciones no es una matriz de identidad y es factible realizar el análisis factorial. Así, se procedió a realizar un AFE mediante el método de extracción 
de "factorización del eje principal" debido a que los factores mostraron correlaciones entre sí mayores de 0.20 , véase tabla 2 .

Tabla 2.

Matriz de correlaciones interfactores

\begin{tabular}{cccccc}
\hline Factor & 1 & 2 & 3 & 4 & 5 \\
\hline 1 & 1.00 & & & & \\
2 & .42 & 1.00 & & & \\
3 & .57 & .30 & 1.00 & & \\
4 & .65 & .33 & .58 & 1.00 & \\
5 & .51 & .40 & .34 & .28 & 1.00 \\
\hline
\end{tabular}

Fuente: elaboración propia.

El método de Kaiser-Guttman, reteniendo aquellos factores con autovalores mayores que 1 , sugirió cinco factores. Se reportaron los ítems con cargas superiores a .40 , dichas cargas oscilaron entre .41 y .76. Además, se mantuvieron aquellos ítems con mayor coherencia teórica dentro del factor y se excluyeron los ítems 2, 7, 11, 16, 21 y 27, los cuales tenían cargas inferiores a .40. Lo anterior, sin afectar la confiabilidad de las subescalas y el mínimo de 3 ítems por factor, según las recomendaciones de Campo-Arias, Herazo y Oviedo (2012). En la tabla 3 se observa la nueva versión de 17 ítems, así como su respectiva distribución de cargas factoriales en cada factor.

Como se puede apreciar en la tabla 3, el factor con mayor porcentaje de varianza y mayor número de ítems es Logro/estatus. Basado en los autovalores iniciales, el porcentaje de varianza total explicado es de $64 \%$.

Análisis de confiabilidad de IRMH-M. Se obtuvieron medidas de consistencia interna del instrumento adecuadas, tanto por subescalas, como para el total (Cronbach $=.88)$ y (Omega de McDonald $=.90)$, las cuales mostraron que la escala posee una alta confiabilidad. La consistencia interna por subescalas se estimó a través de los factores encontrados en el AFE; se encontraron niveles de confiabilidad adecuados con valores de Alfa de Cronbach (a) y Omega de McDonald entre .70 y .79 (véase tabla 3). También se encontró que en todos los ítems la correlación ítem total corregida fue superior a .30, lo cual indica que los ítems del IRMH-M exhiben una adecuada homogeneidad, variando desde un mínimo de $r=.33$ a un máximo de $r=.65$. Además, se obtuvo que todos los ítems contribuyen a la confiabilidad, ya que al eliminar alguno de ellos la confiabilidad no aumenta.

Tabla 3.

Análisis factorial exploratorio y confiabilidad por subescalas del IRMH-M

\begin{tabular}{lcccc}
\hline & \multicolumn{5}{c}{ Factor } \\
\cline { 2 - 5 } & \multicolumn{5}{c}{$\begin{array}{c}3 \\
\text { Logro/estatus }\end{array}$} & $\begin{array}{c}2 \\
\text { Agresión }\end{array}$ & $\begin{array}{c}\text { Actitudes } \\
\text { hacia los homo- } \\
\text { sexuales }\end{array}$ & $\begin{array}{c}\text { Evitación } \\
\text { conductas } \\
\text { femeninas }\end{array}$ & $\begin{array}{c}5 \\
\text { Actitudes } \\
\text { hacia el sexo }\end{array}$ \\
\hline Ítem 1 & .69 & & \\
Ítem 2 & .54 & & \\
Ítem 3 & .45 & & \\
Ítem 4 & .44 & & \\
Ítem 5 & .42 & & & \\
Ítem 6 & & .76 & & \\
Ítem 7 & & .69 & & \\
Ítem 8 & & .58 & & \\
Ítem 9 & & & & \\
Ítem 10 & & & & \\
\hline
\end{tabular}




\begin{tabular}{|c|c|c|c|c|c|c|}
\hline & & \multicolumn{5}{|c|}{ Factor } \\
\hline & & $\begin{array}{c}1 \\
\text { Logro/estatus }\end{array}$ & $\begin{array}{c}2 \\
\text { Agresión }\end{array}$ & $\begin{array}{c}3 \\
\text { Actitudes } \\
\text { hacia los homo- } \\
\text { sexuales }\end{array}$ & $\begin{array}{c}4 \\
\text { Evitación } \\
\text { conductas } \\
\text { femeninas }\end{array}$ & $\begin{array}{c}5 \\
\text { Actitudes } \\
\text { hacia el sexo }\end{array}$ \\
\hline Ítem 11 & & & & .56 & & \\
\hline Ítem 12 & & & & & .70 & \\
\hline Ítem 13 & & & & & .65 & \\
\hline Ítem 14 & & & & & .44 & \\
\hline Ítem 15 & & & & & & .63 \\
\hline Ítem 16 & & & & & & .60 \\
\hline Ítem 17 & & & & & & .41 \\
\hline $\begin{array}{l}\% \text { varianza cor } \\
\text { explicada por }\end{array}$ & ún & 34.85 & 9.33 & 7.35 & 6.44 & 5.64 \\
\hline \multirow[b]{2}{*}{ Confiabilidad } & $\begin{array}{l}\text { Alfa de } \\
\text { Cronbach }\end{array}$ & .79 & .76 & .70 & .72 & .71 \\
\hline & $\begin{array}{l}\text { Omega de } \\
\text { McDonald } \\
\text { (IC 95\%) }\end{array}$ & $.79[0.76,0.82]$ & $.76[0.72,0.79]$ & $.70[0.64,0.75]$ & $.72[0.67,0.76]$ & $.71[0.66,0.75]$ \\
\hline
\end{tabular}

Fuente: elaboración propia.

Nota: IC Intervalo de confianza.

No obstante, y dado que la regla de Kaiser Gutman tiende a sobreestimar o subestimar el número de factores, se decidió confirmar el número de factores obtenidos en el AFE mediante AFC. La tabla 4

Tabla 4.

Análisis factorial confirmatorio del IRMH-M en su versión de 30 y 17 ítems reducida

\begin{tabular}{|c|c|c|c|c|c|c|c|c|c|c|}
\hline Modelo & S-B $x^{2}$ & ggl & $\begin{array}{l}\text { Modelo } \\
\text { AIC }\end{array}$ & NNFI & CFI & GGFI & AGFI & SRMR & RMSEA & IC90\%RMSEA \\
\hline $\begin{array}{l}\text { Cinco factores } \\
\text { Versión } 30 \text { ítems, } \\
\text { (Toro \& Varas, 2003) }\end{array}$ & 1936.39 & 395 & 2076.39 & .95 & .95 & .97 & .96 & .07 & .08 & $\begin{array}{c}(0.077 ; \\
0.084)\end{array}$ \\
\hline $\begin{array}{l}\text { Cinco factores } \\
\text { (Versión reducida } \\
17 \text { ítems) }\end{array}$ & 343.29 & 1109 & 431.29 & .97 & .98 & .99 & .98 & .05 & .06 & $\begin{array}{c}(0.053 ; \\
0.067)\end{array}$ \\
\hline $\begin{array}{l}\text { Cinco factores y un solo } \\
\text { factor de segundo orden } \\
\text { (Versión reducida } \\
17 \text { ítems) }\end{array}$ & 355.84 & 1114 & 433.84 & .97 & .98 & .98 & .98 & .05 & .06 & $\begin{array}{c}(0.053 ; \\
0.067)\end{array}$ \\
\hline
\end{tabular}

Fuente: elaboración propia.

Nota: Método de estimación: DWLS. Todos los $x^{2}: \mathrm{p}<0.001$. IC Intervalo de confianza. S-B $x^{2}$ : Chi cuadrado de Satorra Bentler; gl: Grados de libertad; RMSEA: Root Mean Square Error of Approximation; AIC: Akaike Index; CFI: Comparative Fit Index; AGFI: Adjusted Goodness of Fit Index muestra los resultados del AFC para el modelo pentactorial de 30 ítems del cual se partió y la versión reducida nueva compuesta por 17 ítems. 


\section{Validez discriminante del IRMH-M.}

Se comparó el puntaje total obtenido en el IRMH-M de acuerdo con el nivel educativo encontrando diferencias significativas $\left(x_{(7)}^{2}=34.9, p<.001\right)$ lo cual, indicó que a menor nivel educativo mayor adherencia a los roles de masculinidad hegemónica. Por su parte, al realizar la comparación entre el puntaje total obtenido en el IRMH-M de acuerdo a la ocupación, se encontraron diferencias significativas $\left(x^{2}(7)=27.14, p<.001\right)$ indicando que existe un arraigo diferente a los roles tradicionales de masculinidad en relación a la ocupación del participante. En cuanto a los tres rangos de edad, la prueba permitió establecer diferencias significativas $\left(x^{2}{ }_{(2)}=7.13\right.$, $p=.028$ ) siendo mayor la adherencia a los roles de masculinidad a mayor edad. No obstante, las diferencias fueron significativas sólo entre el grupo de 18 a 25 y el grupo de 36 a 65 años.

Con relación a nivel socioeconómico, se tomaron los niveles con mayor número de participantes (estratos 1 a 4). Los hallazgos también fueron estadísticamente significativos $\left(x^{2}{ }_{(3)}=11.81, p=.008\right)$, siendo mayor la adherencia a los roles de masculinidad en el estrato uno en comparación con el estrato dos. Entre los estratos dos a cuatro, la tendencia fue creciente, a mayor estrato, menor fue la adherencia a los roles de masculinidad. Respecto a estado civil y orientación sexual, no se encontraron diferencias significativas en cuanto a roles de masculinidad hegemónica.

\section{Discusión y conclusión}

El objetivo del presente estudio fue analizar las propiedades psicométricas del IRMH-M en población masculina adulta residente en Tunja (Colombia). Los datos encontrados reflejan que el IRMH-M presenta propiedades psicométricas adecuadas tanto en los niveles de confiabilidad de las distintas dimensiones como una muy buena validez de constructo. Igualmente, el constructo discrimina adecuadamente entre algunas variables como nivel académico y ocupación.

El AFE encontró cinco factores correlacionados entre sí, indicando que los factores son parte de una realidad teórica común llamada masculinidad he- gemónica. Dichos factores estuvieron compuestos por ítems con pesos factoriales mayores a .40 , conforme a rigurosos estándares exigidos (MacCallum, Widaman, Zhang \& Hong, 1999; Williams, Brown \& Onsman, 2010). En el presente estudio, cada factor estuvo compuesto por un número adecuado de ítems (mayor o igual que tres) y hubo correspondencia entre la agrupación de los ítems según los factores donde se esperaba que cargaran. Para hacer una comparación, ver la versión de Levant et al. (1996) que presentan los ítems completos del inventario.

Con respecto al AFC, el índice GFI indica cuánto mejor se ajusta el modelo a los datos comparados con la ausencia de cualquier modelo. Así mismo, indica la varianza común entre el modelo propuesto y los datos. Es bueno si es igual o superior a 0,95; con base en lo encontrado, el ajuste es muy bueno. Lo mismo ocurre con los índices NNFI y CFI.

Para el caso de RMSEA, se presenta un buen ajuste si es igual o inferior a 0,06 (Hu \& Bentler, 1999). En efecto, en ambos modelos de la versión de 17 ítems, los índices RMSEA son muy buenos. SRMR representa el valor residual promediado entre la matriz de varianzas-covarianzas del modelo propuesto y la matriz de varianzas-covarianzas de la matriz de datos. Suele exigirse que sea igual o inferior a .06 (Hu \& Bentler, 1999), por lo que, para ambos modelos, este índice está también dentro de los límites exigidos.

En general, el modelo de cinco factores es mejor al modelo de cinco factores más uno de segundo orden, ya que el valor del índice AIC es más bajo. De las dos versiones de 30 y 17 ítems, la versión reducida resulta con mejores índices de ajuste. Es de notar que el método de extracción del AFC aplicado es DWLS (Diagonally weighted least squares) y este método es recomendado cuando no se cumple la normalidad multivariada (Jöreskog, Sörbom, Totit \& Du toit, 2001; Morata-Ramírez et al., 2015).

El índice de consistencia interna obtenido por Toro-Alfonso y Varas-Díaz (2003) mediante Alfa de Cronbach fue de .90 , el presente estudio encontró una cifra similar (.88). La confiabilidad por subescalas del presente estudio nunca fue inferior de .70 en ninguno de los dos índices, Alfa y Omega. Así, el presente estudio encontró confiabilidad de las subescalas 
superior a estudios previos, (confróntese con Levant \& Majors, 1995; Levant et al.,1996; Levant, et al., 2003). Además, el presente estudio añade fortaleza a los resultados de confiabilidad, puesto que se informa el Omega de McDonald con su respectivo intervalo de confianza al 95\%. Históricamente, no ha habido una tradición importante en corroborar la precisión con la cual está midiendo la prueba mediante varios índices, ya que la mayor parte de los estudios sólo informan el Alfa de Cronbach, el cual tiene serias deficiencias (Dunn et al., 2014).

Validar una prueba psicológica no solo consiste en buscar realizar un análisis factorial y la consistencia interna adecuada; también es importante identificar que el instrumento discrimine según las diversas subpoblaciones a la que fue aplicado. En cuanto al nivel académico y ocupación, se encontraron diferencias significativas. No obstante, no se encontró literatura previa que permitiera contrastar dicho hallazgo.

Con relación a la edad, el presente estudio encontró diferencias significativas entre distintos grupos etarios no adyacentes, pero no entre grupos adyacentes. Dicho hallazgo es consistente con los resultados de Martínez-Gómez et al. (2012) quienes no hallaron diferencias estadísticamente significativas en rasgos de masculinidad feminidad entre adolescentes y adultos jóvenes colombianos. Específicamente, nuestro estudio encontró diferencias entre dos rangos, adultos jóvenes y adultos mayores de 36 años, siendo los roles de masculinidad superiores a mayor edad. Consistente con lo anterior, Rizvi (2000) (citado en Levant \& Richmond, 2007) encontró que, a mayor edad, se presenta una mayor adherencia a los roles de masculinidad hegemónica. Es posible que existan diferencias intergeneracionales y no entre etapas del ciclo de vida.

Asimismo, los estudios de Levant y Richmond (2007) y Bard (2016) hallaron que a menor ingreso y por tanto menor estrato socioeconómico, menor arraigo a los roles de masculinidad hegemónica. Este resultado es consistente con lo encontrado en el presente estudio entre los estratos dos a cuatro, que mostraron dicha tendencia. Sin embargo, no es posible asegurar que las diferencias sean significativas debido a que la prueba $\mathrm{H}$ de Kruskal Walis no permite establecer entre qué estratos específicamente hubo diferencias. En consecuencia, el IRMH-M arroja resultados consistentes, ya que se espera que a mayor estrato mejor es el nivel educativo alcanzado, ambas variables asociadas a roles de masculinidad más bajos. Esto, posiblemente porque mayor educación e ingresos también implican mayor flexibilidad en los roles de género (Levant et al., 2003).

Conforme a lo esperado, el presente estudio arrojó una estructura pentafactorial, que replica la mayor parte de los ítems de 5 de las 7 subescalas de Levant, Wu y Fisher (1996), evidenciando semejanzas en cómo los hombres construyen su masculinidad en diversos contextos (Estados Unidos y Colombia). Los resultados muestran que el IRMH-M versión reducida compuesta por 17 ítems, brinda datos claros y precisos para la población masculina residente en Tunja (Colombia) que contribuyen con una mejor comprensión de la vivencia de la masculinidad en esta zona del país.

El uso de los dos métodos AFE y AFC usados en el artículo es una ganancia en la comprensión del atributo y constituye un aporte a la exploración del IRMH-M en un contexto latinoamericano como Colombia y en la confirmación de la estructura subyacente de la masculinidad hegemónica. Cada uno de los métodos es distinto y tienen un lugar importante en la psicometría. El primero de explorar y construir la teoría y el segundo confirmar la estructura factorial subyacente previamente formulada y establecida en un contexto, es decir, entender el nivel de ajuste de los datos a la teoría, véase, Lloret-Segura, Ferreres-Traver, Hernández-Baeza y Tomás-Marco (2014). Como lo sugiere Gómez, Lazcano y Salazar (2007), la validación de un instrumento para un contexto determinado permite conocer de una mejor manera el comportamiento de las personas y contribuye a pensar en la mejor intervención para el caso. Así mismo, es esencial contar con instrumentos culturalmente validados, dado que los reactivos y términos empleados originalmente responden a un determinado contexto.

Dentro de las recomendaciones para futuras investigaciones, se sugieren más estudios con variadas poblaciones en los que se evalúen, la raza, el sexo, 
la generación, la orientación sexual, nivel de estudios, ocupación y estado civil, entre otros, que permitan confirmar o refutar los hallazgos del presente estudio. Otra sugerencia es la búsqueda de un criterio adecuado que permita establecer la validez convergente de la prueba y la comparación de los resultados entre teoría de respuesta al ítem TRI y teoría clásica de los test TCT.

Es muy probable que conforme cambian las características sociales, económicas y culturales de nuestra sociedad, muchos de los ítems deban ser reemplazados y adaptados. Esto en consonancia con el modelo teórico socio-construccionista de masculinidad que sustenta la noción del constructo masculinidad hegemónica, en el que la masculinidad no es atemporal ni universal sino contextual y dinámica (Hernández, 2008). Se sugiere además que en investigaciones futuras el constructo sea validado en mujeres.

Se concluye que el IRMH-M posee propiedades psicométricas adecuadas y este inventario puede usarse en investigaciones que indaguen sobre el constructo y su posible influencia en la salud de los hombres. También puede usarse por profesionales de la salud y la clínica interesados en intervenir en la reducción de los roles tradicionales de masculinidad que afectan la salud mental de quienes reportan alta adherencia a los roles de masculinidad hegemónica, que en la actualidad se ha convertido en el motor de la violencia asociada al género.

\section{Referencias}

Bard, G. (2016). Aferrarse o soltar privilegios de género: Sobre masculinidades hegemónicas y disidentes. Península. 11, 2, 101-122.

Barra, E. (2004). Validación de un inventario de rol sexual construido en Chile. Revista Latinoamericana de Psicología, 36, 97-106.

Bem, S. (1981). Gender schema theory: A cognitive account of sex typing. Psychological Review, 88, 354-364.

Brannon, R. \& Juni, S. (1984). A scale for measuring attitudes about masculinity. Psychological Documents, 14(1), ID 2612
Brown, J. M. (2002). Statistical analysis of a measure of adolescent gender ideology, the Male Role Norms Inventory-Adolescent: A Pilot Study. Unpublished doctoral directed study, Nova Southeastern University.

Burin, M. \& Meler, I. (2000). Género.Una herramienta teórica para el estudio de la subjetividad masculina. En M. Burin, \& I. Meler (Eds.), Varones: Género y subjetividad masculina (1st ed., pp. 21-70). Argentina: Paidós.

Bustamante, W. (2013). Masculinidad y homofobia. El control de la sexualidad del varón en la construcción del Estado colombiano. Sociedad y Economía, 24, 159-182.

Campo-Arias, A., Herazo, E. \& Oviedo, H. (2012). Análisis de factores: fundamentos para la evaluación de instrumentos de medición en salud mental. Revista Colombiana de Psiquiatría, 41(3), 659-671.

Cruz, J. (2007). Actitudes de los heterosexuales hacia los homosexuales como fenómeno social: una revisión conceptual. (Tesis inédita) Universidad Nacional de Colombia. Facultad de Ciencias Humanas. Colombia.

Davis, F. (1987). Antecedents and consequents of gender role conflict: An empirical test of sex role strain analysis (Doctoral dissertation).Ohio State University, United States of America. Recuperado de https://etd.ohiolink.edu/ rws_etd/document/get/osu1392046090/inline

David, D. \& Brannon, R. (1976). The male sex role: Our culture's blueprint for manhood, and what it's done for us lately. In D. David \& $\mathrm{R}$ Brannon (Eds.), The fortynine percent majority: The male sex role (pp. 1-48). Reading, MA: Addison-Wesley.

Dunn, T. J., Baguley, T., \& Brunsden, V. (2014), From alpha to omega: A practical solution to the pervasive problem of internal consistency estimation. British Journal of Psychology, 105: 399-412. DOI: 10.1111/bjop.12046

Estrada, J. H. \& Sánchez-Alfaro, L. A. (2011). Las violencias de género como problema de salud 
pública: una lectura en clave Bioética. Revista Colombiana de Bioética, 6(1), 37-61.

Faur, E. (2004). Masculinidades y desarrollo social: Las relaciones de género desde la perspectiva de los hombres. Recuperado de http://www. unicef.org/colombia/pdf/masculinidades-pdf

Gómez, M., Lazcano, M., \& Salazar B. (2007). Validación del instrumento: Afrontamiento y procesos de adaptación de Roy en pacientes Mellitus Tipo II. Aquichan, 8(1), 116-125.

Good, G. E. \& Mintz L. B. (1990). Gender role conflict and depression in college men: Evidence for compounded risk. Journal of Counseling and Development, 69(1), 17-21.

Hernández, O. (2008). Estudios sobre masculinidades. Aportes desde américa latina. Revista de Antropología Experimental, 8(5), 67-73.

Hu, L. \& Bentler, P. M. (1999). Cut-off criteria for fit indices in covariance structure analysis: conventional criteria versus new alternatives. Structural Equation Modeling $A$ Multidisciplinary Journal, 6(1), 1-55. DOI: 10.1080/10705519909540118

Jakupcak, M., Lisak, D., \& Roemer, L. (2002). The role of masculine ideology and masculine gender role stress in men's perpetration of relationship violence. Psychology of Men and Masculinity, 3(2), 97-106. DOI: 10.1037/1524-9220.3.2.97

Jewkes, R., Morrel., R., Hearn, J., Lundqvist, E., Blackbeard, D., ... Gottzén, L. (2015). Hegemonic masculinity: combining theory and practice in gender interventions. Culture, Health \& Sexuality, 17(2), 112-127. DOI: 10.1080/13691058.2015.1085094

Jöreskog, K. G., Sörbom, D., Du Totit, S., \& Du toit, M. (2001). LISREL 8: New statistical features. Third priting with revisions. Chicago: Scientific Software International.

Kerns, J. G. \& Fine, M. A. (1994). The relation between gender and negative attitudes toward gay men and lesbians: Do gender role attitudes mediate this relation? Sex Roles, 31, 297-307.

Lara, M. A. (1993). Inventario de masculinidad y feminidad. Ciudad de México:Manual Moderno.

Levant, R. F., Cuthbert, A. C., Richmond, K., Sellers, A., Matveev, A. ... Heesacker, M. (2003). Masculinity ideology among Russian and U.S. young men and women and its relationship to unhealthy lifestyle habits among young Russian men. Psychology of Men and Masculinity,4, 26-36.

Levant, R. F., Graef, S. T., Smalley, K. B., Williams, C., \& McMillan, N. (2008). The evaluation of the Male Role Norms Inventory-Adolescent (MRNI-A). Thymos: Journal of Boyhood Studies, 2(1), 46-59.

Levant, R. F., Hall, R. J., \& Rankin, T. J. (2013). Male Role Norms Inventory-Short Form (MRNISF): Development, confirmatory factor analytic investigation of structure, and measurement invariance across gender. Journal of Counseling Psychology, 60, 228-238. DOI: 10.1037/a0031545

Levant, R. F. \& Majors, R. G. (1995). An investigation into variations in the construction of the male gender role among young African American and European American women and men. Journal of Gender, Culture, and Health, 2, 33-43.

Levant, R. F. \& Richmond, K. (2007). A review of research on masculinity ideologies using the Male Role Norms Inventory. The Journal of Men's Studies, 15(2), 130-146. DOI: 10.3149/ jms.1502.130

Levant, R. F., Smalley, K. B., Aupont, M., House, A. T., Richmond, K. \& Noronha, D. (2007). Initial Validation of the Male Role Norms InventoryRevised (MRNI-R). The Journal of Men's Studies, 15(1), 83-100. DOI: 10.3149/jms.1501.83

Levant, R. F., Wu, R., \& Fischer, J. (1996). Masculinity ideology: A comparison between U.S. and Chinese young men and women. Journal of Gender, Culture and Health, 1, 3217-220. 
Lloret-Segura, S., Ferreres-Traver, A., HernándezBaeza, A., \& Tomás-Marco, I. (2014). El Análisis Factorial Exploratorio de los Ítems: una guía práctica, revisada y actualizada. Anales de Psicología, 30(3), 1151-1169. DOI: 10.6018/ analesps.30.3.199361

Lozano, I. \& Rocha, T. (2011). La homofobia y su relación con la masculinidad hegemónica en México. Revista Puertorriqueña de Psicología, 22, 101-121.

MacCallum, R. C. , Widaman, K. F., Zhang, S., \& Hong, S. (1999). Sample size in factor analysis. Psychological Methods, 4(1), 84-99.

Martínez-Gómez, J., Guerrero-Rodríguez, S. M., \& Rey-Anacona, C.A. (2012). Evaluación de la validez de constructo y la confiabilidad del inventario de masculinidad y femineidad en adolescentes y adultos jóvenes colombianos. Avances en Psicología Latinoamericana, 30(1), 170-181.

Ministerio de Salud. (1993). Resolución 8430 de 1993: Normas científicas técnicas y administrativas para la investigación en Salud. Bogotá: Minsalud.

Montero, I. \& León, O. (2007). Psychology, A guide for naming research studies in Psychology. International Journal of Clinical and Health Psychology, 7(3), 847-862.

Morata-Ramírez, M. A., Holgado-Tello, F. P., BarberoGarcía, I., \& Mendez, G. (2015). Análisis factorial confirmatorio: recomendaciones sobre mínimos cuadrados no ponderados en función del error Tipo I de Ji-Cuadrado y RMSEA. Acción Psicológica, 12(1), 79-90. DOI: 10.5944/ ap.12.1.14362
Organización Panamericana de la Salud (2010). Género y salud: una Guía Práctica para la Incorporación de la Perspectiva de Género en Salud. Recuperado de http://www.paho.org/ hq/dmdocuments/2010/manualFinal.pdf?ua=1

Scott-Samuel, A., Stanistreet, D., \& Crawshaw, P. (2009). Hegemonic masculinity, structural violence and health inequalities. Critical Public Health, 19, 3-4, 287-292. DOI: 10.1080/09581590903216420

Toro-Alfonso, J. \& Varas-Díaz, N. (2003). Inventario de normas de roles masculinos modificado. Manuscrito inédito. Universidad de Puerto Rico, Río Piedras.

Toro-Alfonso, J., Walters-Pacheco, K. Z., \& Sánchez, I. (2012). El cuerpo en forma: Masculinidad, imagen corporal y trastornos en la conducta alimentaria de atletas varones universitarios. Acta de Investigación Psicológica, 2(3), 842-857.

Torres-Pagán, L. \& Toro-Alfonso, J. (2017). Hegemonic masculinity as a key factor on health beliefs and seeking help in Puerto Rican men with hypertension: A qualitative study. Revista Puertorriqueña de Psicología, 28(1), 134-147.

Vázquez, V. \& Castro, R. (2009). Masculinidad hegemónica, violencia y consumo de alcohol en el medio universitario. Revista Mexicana de Investigación Educativa, 14(42), 701-719.

Williams, B., Brown, T., \& Onsman, A. (2010). Exploratory factor analysis: A five-step guide for novices. Australasian Journal of Paramedicine, 8(3), 1-13. 
ANEXO

Composición de los ítems del IRMH-M

\section{ITEMS}

1. El trabajo doméstico es trabajo de mujeres.

2. Empleos como bombero y electricista deben ser para hombres.

3. Los niños deben preferir jugar con camiones en vez de jugar con muñecas.

4. Los niños no deben tirar las canicas (bolitas, maras) como las niñas.

5. Un hombre debe evitar en todo momento cargar la cartera de su esposa.

6. Es afeminado que un hombre se pinte las uñas con esmalte transparente.

7. Uno de los peores insultos dirigidos a un hombre es llamarlo "maricón".

8. Un hombre no debe continuar la amistad con otro hombre si descubre que éste es homosexual.

9. Hay ciertos temas que los hombres no deben hablar con otros hombres.

10. Es decepcionante enterarse que un atleta famoso es homosexual.

11. Los niños deben ser motivados a encontrar formas de demostrar su fuerza física.

12. Un hombre que no le guste la aventura, no es muy atractivo para las mujeres.

13. Un hombre debe levantarse para investigar cuándo hay un ruido extraño en la casa durante la noche.

14. Es importante para un hombre asumir riesgos aunque pueda ser herido.

15. Un hombre debe ser fuerte en los momentos difíciles.

16. Si es necesario, un hombre debe sacrificar sus relaciones personales para avanzar en su carrera profesional

17. En un grupo, le corresponde a los hombres organizar las cosas y emprender la marcha.

18. Un hombre debe hacer lo que sea para ser admirado y respetado.

19. Está bien que un hombre compre un carro deportivo, si lo desea aunque se sobrepase en su presupuesto.

20. Un hombre debe ser siempre el proveedor principal en su familia.

21. El hombre debe tratar de ganar en cualquier deporte que participe.

22. En situaciones que involucran dinero, la decisión final le corresponde a los hombres.

23. El hombre debe estar siempre dispuesto a tener sexo.

24. Es importante que un hombre sea bueno en la cama.

25. Los hombres deben tener siempre la iniciativa en el sexo.

26. Un hombre no debe preocuparse sobre el control de la natalidad.

27. Para un hombre, el sexo debe ser espontáneo, en vez de una actividad planificada.

28. Para un hombre las caricias son el primer paso hacia el sexo.

29. Abrazar y besar deben llevar siempre a la penetración.

30. No vale la pena tener sexo a menos que pueda alcanzar un orgasmo.

Nota: en negrita: versión de 30 ítems Toro y Varas (2003). En cursiva, versión 17 ítems de la versión reducida final. 